\title{
Clinical Trial Risk in Type-2 Diabetes: Importance of Patient History
}

\author{
Emmanuel O. Aiyere ${ }^{1}$, Jay Silverberg ${ }^{2}$, Safina $\mathrm{Ali}^{3}$, and Jayson L. Parker ${ }^{1}$ \\ ${ }^{1}$ Department of Biology, University of Toronto at Mississauga, Toronto, ON, Canada; ${ }^{2}$ Sunnybrook Health Sciences \\ Centre, Toronto, ON; ${ }^{3}$ Laboratory Medicine and Pathobiology, University of Toronto, Toronto, ON, Canada
}

Received, March 26, 2014; Revised, July 7, 2014; Accepted, August 1, 2014; Published, August 25, 2014

\begin{abstract}
Purpose. To determine the risk of clinical trial failure for drugs developed for type-2 diabetes. Methods. Drugs were investigated by reviewing phase I to phase III studies that were conducted between 1998 and February 2013. The clinical trial success rates were calculated and compared to the industry standard. The drugs were classified into GLP-1 receptor agonists, DPP-4 inhibitors, SGLT-2 inhibitors and "Other". The exclusion criteria for drugs in this study: Drugs that were started in phase I studies prior to January 1998 for this indication and drugs whose primary indications were not for the control of blood glucose levels. Results. Data was extracted from clinicaltrials.gov; there were a total of 131 drug candidates that fit our specified criteria, of which 8 received FDA approval. The cumulative success rate for molecules developed for type-2 diabetes is $10 \%$. Small molecules were more successful than biologics. A strong disparity was observed in phase III, with studies that utilised treatment naïve patients having a $40 \%$ success rate, compared to an $83 \%$ success rate in patients who have had previous anti-hyperglycemic exposure. Conclusions. 1 in 10 drugs that enter clinical testing in this disease will be approved. The DPP-4 inhibitor class of drugs had the highest success rate of all drug classes with a 63\% cumulative success rate; while treatment naïve patients carried the greatest clinical trial risk. Keywords: Clinical trials, Type-2 diabetes, Drug development, Clinical trial risk.
\end{abstract}

This article is open to POST-PUBLICATION REVIEW. Registered readers (see "For Readers") may comment by clicking on ABSTRACT on the issue's contents page.

\section{INTRODUCTION}

Currently, diabetes is viewed by many as an epidemic due to its enormous rise in prevalence, which has tripled since 1985 (1). Key drivers include obesity and physical inactivity (2), and the concern amongst healthcare officials has become further emboldened by the cost of intervention; reaching $\$ 174$ billion US per annum (3). As it stands, $95 \%$ of these diabetes cases are classified as type-2 or "insulin resistant diabetes (4). When treating a patient, if lifestyle changes do not result in adequate blood-sugar control within 3 to 6 months, pharmaceutical intervention usually commences with metformin (5). If blood glucose control does not improve, a physician is likely to prescribe metformin in addition to one other pharmaceutical agent (e.g., sulfonylurea or meglitinide, thiazolidinedione, dipeptidyl peptidase4 inhibitor, glucagon-like peptide- 1 receptor agonists (GLP-1), sodium-dependent glucose transporter - 2 Inhibitor (SGLT-2), and rarely an alpha glucosidase inhibitor as well as basal insulin). Where a patient's hyperglycemia cannot be controlled, a physician is usually faced with placing the patient on a 3-drug combination, or utilizing basal insulin (6).

Up to $17 \%$ of patients who are initiated on metformin monotherapy fail to reach their glycemic target within 1 year (7).With the FDA approval of canagliflozin (an SGLT2 inhibitor) 2013 (8), there are now 3 new drug classes that physicians can choose from when determining the course of treatment. The new drug classes are GLP-1 receptor agonists, DPP-4 inhibitors and SGLT-2 inhibitors.

GLP-1 receptor agonists mimic the action of endogenous GLP-1 and are resistant to DPP-4, which is an enzyme that deactivates GLP-1 (9).

Correspondence Author: Jayson Parker, Department of Biology, University of Toronto Mississauga, 3359 Mississauga Road, Mississauga, ON L5L 1C6 Canada, Office DV2034A. Email: Jayson.parker@utoronto.ca 
Similarly, DPP-4 inhibitors work by inhibiting the activity of DPP-4 enzyme in the blood, therefore increasing the half-life of GLP-1 upon secretion. With respect to SGLT-2, these molecules work by inhibiting re-absorption of glucose by the kidney. In doing so, increased blood glucose is excreted through the urine. This unique mechanism of action has multiple functionality which allows the reduction of blood glucose levels, while at the same time leading to weight loss and blood pressure reduction (10).

With increasing research efforts to combat type-2 diabetes, it is important to quantify the clinical trial risk associated with drug development. In order to do this, we have evaluated clinical studies in the USA pertaining to type- 2 diabetes, and quantified the probability of a drug successfully advancing to the next phase of clinical trials and factors that may impact that risk estimate. The methodology used in this paper has been applied to other diseases areas (11-16).

\section{METHODS}

\section{Data Source}

Clinical trial data was gathered from www.clinicaltrials.gov, a clinical trial registry that houses publicly and privately funded clinical trials and results of studies conducted in 50 American states and 187 countries. It is backed by Section 801 of the Food and Drug Administration Modernization Act, which enforces penalties for failure to register or report study results.

\section{Study Eligibility}

Phase I, II, or III clinical studies in adults with type-2 diabetes from January 1998 to February 2013 were included in the analysis. Approved drugs with phase I trials conducted before 1998 were excluded from our study. All studies included in this study had to have end points that were relevant to glucose control (eg. HbA1c scores and Fasting Plasma Glucose levels). In order to be aligned with Section 801 of the Food and Drug Administration Amendment Act which states that all publicly and privately sponsored clinical trials must be registered on clinicaltrials.gov, a criteria for inclusion in this study was that all studies must be industrysponsored and have at least one US site. A drug was considered a "line extension" if it had been previously approved for a different indication and subsequently entered testing for type-2 diabetes. Phase I/II trials were considered as phase I, while phase II/III trials were considered as phase II. For this study, we excluded any drug formulation that included insulin.

\section{Clinical Trial Outcome Classification}

Phase I and I/II clinical testing was classified as a "success" if the drug advanced to phase II. Phase II clinical testing was classified as a "success" if the drug advanced to phase III. Phase III clinical testing was classified as a "success" if the US Food and Drug Administration (FDA) approved the compound and it remained on the market as of March 2011.

\section{Classification of Clinical Trial Failure}

Clinical trial failure was separated into clinical and commercial failure. A clinical failure was defined as one where a drug failed to meet its endpoint in phase II or phase III, or had significant safety issues during any of the phases. If a clinical trial was "withdrawn", "terminated" or a drug was withdrawn from the market, it was considered a clinical failure. Commercial failure was defined as a drug program that showed no indications of clinical failure in press releases or conference proceedings, yet there were no signs of further clinical testing of the drug conducted in 2 or more years. Commercial failures could be the result lack of financing, revisions of revenue forecast and competing drug programs. Any drug program that completed trial in the last 2 years, but had no indication of clinical failure was considered "unclassified/unknown" and were not included in the analysis.

\section{Clinical Trial Success Rate}

The clinical trial success rate was calculated by determining the percentage of successful trials out of the total number of trials in a particular phase, as follows:

Success rate for phase $\mathrm{x}=$

(number of drug candidates that successfully completed phase $x$ )

(total number of drugs that completed phase $X$ )

The "number of drug candidates that successfully completed phase $x$ " refers to the number of drug candidates that successfully 
completed phase $x$ and moved on to phase $x+1$ (and/or phase $x+2$ ). The denominator includes the number of drugs that completed phase $X$ but did not move on to phase $X+1$. Cumulative rates refer to the probability of completing the current clinical trial and any preceding clinical trial phase successfully (i.e., the product of probabilities). For example, a drug that is currently in phase III is a success for both phase I and phase II given that those trials were completed in the specified time period of this study.

\section{RESULTS}

After searching clinicaltrials.gov, an initial search yielded a result of 5692 clinical trials. All clinical trial phases for the drugs used included this analysis were registered on clinicaltrials.gov. After removing multiple hits for a single drug, 131 drug candidates met the inclusion criteria for our study. These were molecules that initiated phase I clinical trials between January 1998 and February 2013 in adult patients with type- 2 diabetes being treated with a pharmaceutical agent that used endpoints relevant to hyperglycaemic control. Of these drugs, 48 successfully completed phase I, 11 successfully completed phase II and 8 successfully completed phase III. The approved drugs that fit the criteria outlined in this study were exenatide (approved in 2005) (17), sitagliptin (approved in 2006) (18), saxagliptin (approved in 2009) (19), liraglutide (approved in 2010) (20), linagliptin (approved in 2011) (21), alogliptin (approved in 2013) (22), canagliflozin (approved in 2013) (8) and dapagliflozin (approved in 2014) (23). Four out of these eight molecules were DPP-4 inhibitors (alogliptin, linagliptin, saxagliptin and sitagliptin).

Based on the data collected, the success rates for each phase was calculated and compared to those of industry standards and other therapeutic areas (Table 1). The industry standard is based on previously reported data from a study that encompassed multiple therapeutic areas being investigated by 50 of the largest pharmaceutical firms (by sales) in the US (24). For phases I and II the transition probabilities were lower than those seen for the industry as a whole, while phase III was $1 \%$ above the industry standard (Figure 1). Accordingly, the cumulative success rate for type- 2 diabetes is $10 \%$.
Table 1. The cumulative success rate of clinical trials in multiple therapeutic areas

\begin{tabular}{|c|c|}
\hline Disease area & $\begin{array}{c}\text { Clinical trial } \\
\text { success rate }\end{array}$ \\
\hline Industry standard (24) & $16 \%$ \\
\hline $\begin{array}{l}\text { HER-2 Positive Advanced Metastatic } \\
\text { Breast Cancer (27) }\end{array}$ & $23 \%$ \\
\hline $\begin{array}{l}\text { Moderate to Severe Crohn's disease } \\
\text { (13) }\end{array}$ & $19 \%$ \\
\hline Human immunodeficiency virus (11) & $16.7 \%$ \\
\hline Rheumatoid Arthritis (16) & $16 \%$ \\
\hline $\begin{array}{l}\text { Advanced Metastatic Breast Cancer* } \\
\text { (27) }\end{array}$ & $15 \%$ \\
\hline Non-Small Cell Lung Cancer (12) & $11 \%$ \\
\hline Non-Hodgkin's Lymphoma (14) & $8-11 \%$ \\
\hline Type-2 diabetes & $10 \%$ \\
\hline $\begin{array}{l}\text { Castration Resistant Prostate cancer } \\
\text { (15) }\end{array}$ & $3 \%$ \\
\hline
\end{tabular}

* - Advanced Metastatic Breast Cancer clinical trials that did not use the HER-2 biomarker as a selection criteria

Failures for all phases were classified into commercial and clinical failures. In phase I, 8 trials were classified as being clinical failures; while 41 were considered to be commercial failures. This number decreases significantly in phase II, where 6 of the drugs were halted due to clinical failures, while 11 of the drugs were classified as commercial failures. Finally, phase III had one commercial failure.

We analyzed pass rates based on whether drug candidates were biologics or small molecules. Small molecules showed a success rate of $45 \%$ in phase I, which is much higher than the success rate of biologics (29\%; Figure 2). Both classes of drugs were much lower than the industry rate of $64 \%$. In phase II, biologics fared better than small molecules substantially, with a $55 \%$ success rate in comparison to $32 \%$ for small molecules. In phase III the pattern in phase II reversed; small molecules had a success rate of $71 \%$, while biologics had a $40 \%$ success rate. Based on the above results, the cumulative success rate for small molecules was $10 \%$, while biologics lagged behind with a $6 \%$ cumulative success rate; both well below industry expectations of $16 \%$ (24).

Phase II and III clinical trials were broken down based on their patient history by examining their enrolment criteria: studies using patients previously exposed to an anti-hyperglycaemic agent 
versus studies using patients who were treatment naïve to any previous anti-hyperglycemic drug exposure. Phase I studies were omitted from this analysis because these studies are mainly focused on the pharmacokinetic and pharmacodynamics properties of the molecule in healthy patients. Clinical trials that used patients that had preexposure to other anti-hyperglycemic agents had a higher success rate in both phase II and phase III (Figure 3). In phase II trials, the use of patients that had pre-exposure resulted in a 33\% success rate, compared to $20 \%$ for treatment naïve patients. This disparity was higher in phase III, where pre- exposure resulted in an $83 \%$ success rate, compared to $40 \%$ for clinical trials that used treatment naïve patients.

Success rates for different mechanisms of actions were evaluated (Figure 4). Molecules in the DPP-4 drug class have the highest success rate with a $63 \%$ cumulative success rate. GLP-1 drug class was the second most successful with a $22 \%$ cumulative success rate, while the SGLT2 drug class were the least successful with a cumulative success rate of $19 \%$. As of right now, no drugs that fall under "other" drug classes have been approved.

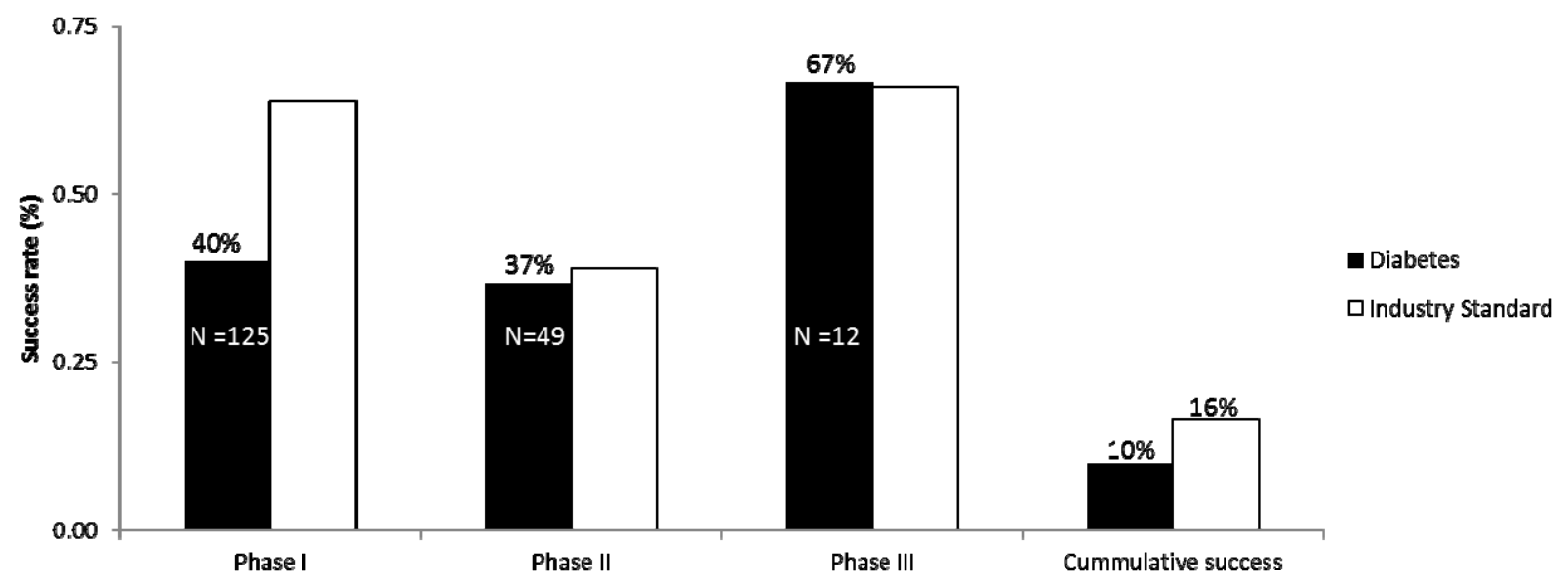

Figure 1. The success rates and cumulative success rates of drug candidates for type-2 diabetes versus Industry standard are illustrated at each phase of clinical trial.

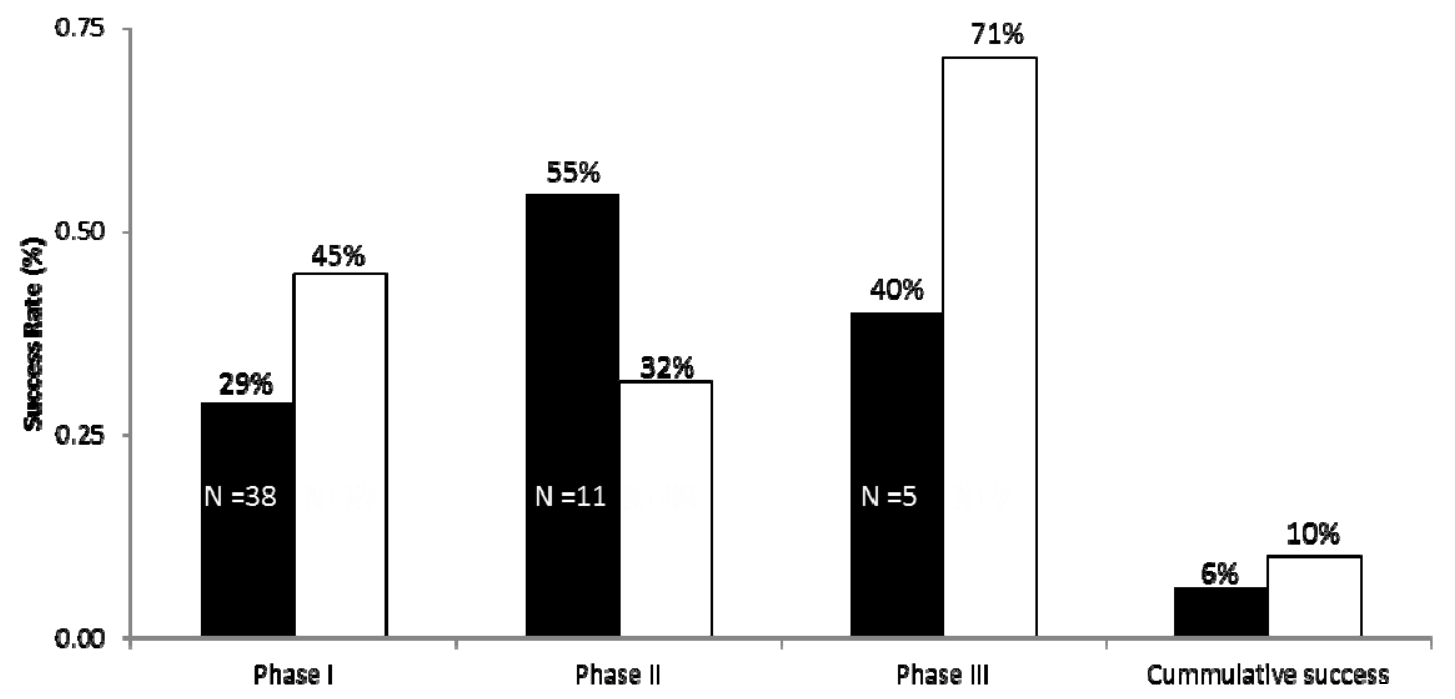

- Blologics

口Small molecules

Figure 2. The success rates of drug candidates based on compound type are illustrated i.e., small molecules versus biologics phase I, II, III and cumulative success rates. 


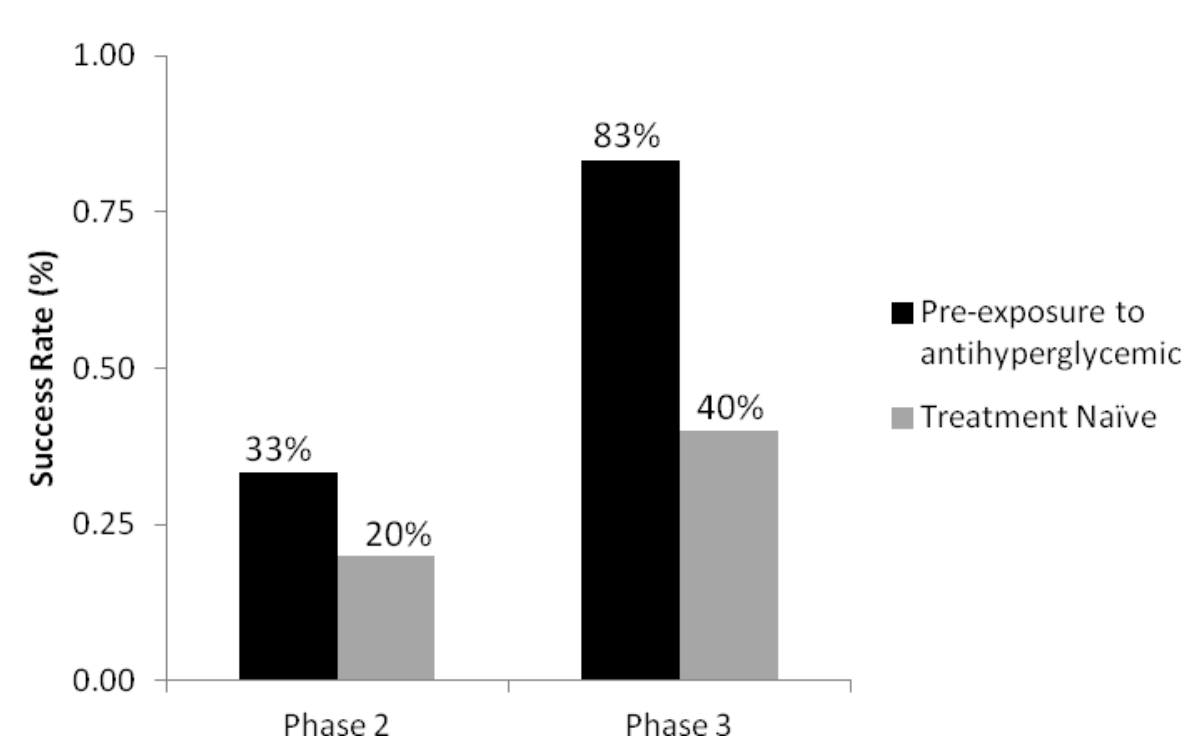

Figure 3. Success rate for pre-exposure versus treatment naïve patients in phase II and II clinical trials.

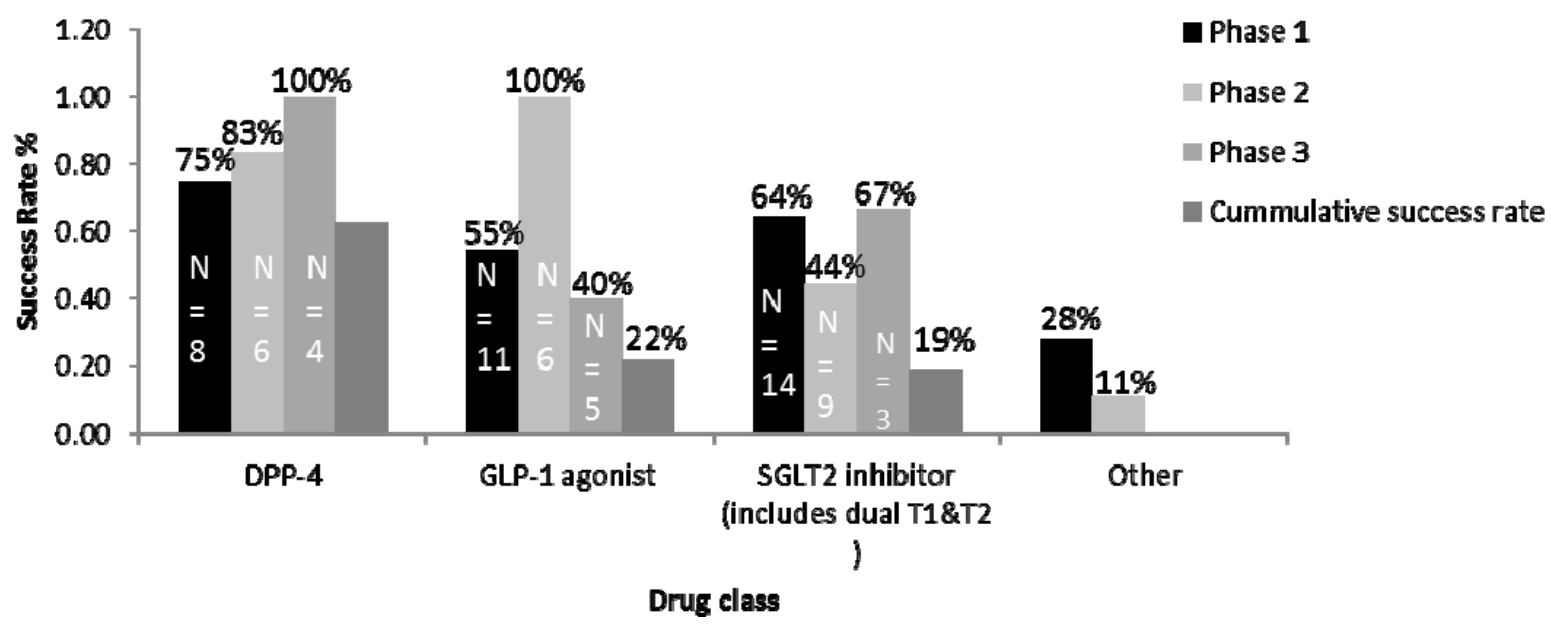

Figure 4. Success rates of different drug classes at all three phases of the clinical trial development process.

\section{DISCUSSION}

In order to quantify the risk of clinical trial failures for molecules undergoing evaluated for the treatment of type- 2 diabetes, we examined clinical trial outcomes. Based on the results of this study, it was determined that molecules in clinical trial for type- 2 diabetes have a cumulative success rate (or probability of US FDA approval) of $10 \%$. This is less than the industry cumulative success rate of $16 \%$. Based on these results, 1 out of 10 molecules initiating phase I clinical trials will eventually be available to patients. The cumulative success rate for type- 2 diabetes molecules are therefore lower than molecules explored for the treatment of human immunodeficiency virus (11), Non-small cell lung cancer (12) and moderate to severe Crohn's disease (13). However, type-2 diabetes drug development shows more promise than clinical trials for castration resistant cancer (15). When evaluated by the type of molecule, small molecules have a $4 \%$ higher success rate than biologics. In addition, 
clinical trials involving patients who have previously undergone treatment with an antihyperglycemic agent were substantially more successful than trials that used treatment naïve patients. When evaluated by drug class, the most successful molecules were molecules in the DPP-4 inhibitor drug class which make up more than 50\% of drugs that successfully gained FDA approval.

The clinical trial risk carried for type-2 diabetes trials was high, considering the cumulative pass rate of $10 \%$. Given this disappointing performance, there is a clear need to identify areas for improvement. The high failure rate of phase I would normally be encouraging, as it is better to experience attrition earlier in clinical testing when costs and patient populations are much smaller compared to later phases. For example, in HIV clinical trials, the best performing drug class in terms of risk (protease inhibitors), also had the lowest pass rate for phase I testing (11). A similar pattern is seen here in the performance of DPP-4 inhibitors. For type-2 diabetes, this could mean phase I is doing a very poor job screening drugs that will be problematic in later testing, or drugs tested in diabetes are inherently at greater risk of failure. As transition probabilities for phase II and phase III are similar to those seen in industry, this would suggest a driving factor for the challenges in clinical trial testing in type- 2 diabetes is due to phase I studies. In this context, it may mean, rather than failing to screen out problematic drugs, phase I studies in diabetes may also be inadvertently screening out potentially promising compounds, which could also contribute to increased clinical trial risk.

When clinical trial success was evaluated by molecule type, it was evident that small molecules and biologics show opposite risk profiles. In the past, many have viewed biologic molecules with more optimism in drug development when compared to small molecules (24), but in our research we have not seen a clear trend, as it appears to vary with the indication (11-16). Based on cumulative success rates, small molecules are more successful in clinical trials for type-2 diabetes. The high number of biologic molecules that fail in phase I might be a result of biologic molecules inducing immunogenicity in patients. According to previous research, a factor influencing immunogenicity could be the route of administration and frequency of administration; proteins administered through the subcutaneous or intradermal route were more likely to induce immunogenicity (25). The risk of immunogenicity is also supported by an observed increase in the incidences of adverse events linked to biologics (26). In other therapeutic areas such as castration resistant cancer (15) and advanced metastatic breast cancer (27), it has been found that the use of biologic response markers, helped to improve clinical trial success rates (28). Such an approach could also be implemented in this therapeutic area to better determine what patient segment a particular therapy will benefit most. In contrast, the high percentage of molecules screened out phase II for small molecules leads to a high success rate in phase III, suggesting that phase II trials of small molecules screened out low potential molecules effectively.

To gain further insight into factors that influence clinical trial success, the impact of patient type was evaluated. Upon analysis, it was evident that enrolment of patients who have previously been exposed to an anti-hyperglycemic agent led to higher clinical trial success. This result supports the step-wise approach physicians currently take when treating patients (5); such a result is surprising because patients who are treatment naive are usually expected to be at an earlier stage of diabetes, therefore they usually have better beta cell reserve. However, as discussed earlier, $17 \%$ of patients fail metformin monotherapy within a year; hence add-on therapies are often utilized. The higher success rate observed in clinical trials that enrol patients who aren't treatment naive further supports current treatment guidelines for the treatment of patients with type-2 diabetes, because most patients typically require an add-on therapy.

Surprisingly, although GLP-1 receptor agonists have been shown to be more effective in lowering body weight, blood glucose and plasma lipids compared to DPP-4 inhibitors in comparative head to head studies (29); our analysis shows DPP4 inhibitors have the highest cumulative success rate. A contributing factor to the success of DPP-4 inhibitors over other drug classes in phase II and III might be the study population utilized. We observed in the DPP-4 inhibitor studies that phase II and III trials had patients that were naïve to previous antihyperglycemic agents, whereas the GLP-1 receptor agonist and SGLT2 inhibitor studies had no treatment naïve patients. This suggests that the 
GLP-1 receptor agonists and SGLT2 inhibitors in phase II and III study patient population had more severe diabetes compared to the patients in the DPP-4 inhibitor studies. As a result, the patients in the GLP-1 receptor agonists and SGLT2 inhibitors studies have had previous exposure to antihyperglycaemic drugs. As treatment guidelines suggest, an additive effect is often sought when treating patients with type-2 diabetes (5), therefore, the use of treatment naïve patients in DPP-4 clinical trials, suggests they're quite often selecting patients at earlier stages of the disease with less severe symptoms and co-morbidities.

Our study has many limitations, which have been discussed in examining other specific disease areas (11-16). The trade-off in examining a specific indication is that we have a much smaller sample size in which to perform our analysis. In addition, we have not conducted third party review of the data but have instead classified trials as successful if they advanced to the next phase of clinical testing. This is an assumption that may warrant further study, but is well outside this initial report. As a result, we cannot by any means see these results as definitive, but as potential indicators we should be aware of. In the classification of clinical failures, some of the studies were simply classified as "terminated" on clinicaltrials.gov. Due to this, we were unable to identify if the studies were terminated a result of safety or efficacy issues.

It is evident that drug development for type-2 diabetes lags behind other diseases areas. There is a cumulative success rate of $10 \%$ in this space; which is well below the $16 \%$ rate of success that previous research has shown to be the clinical approval rate of success of molecules being investigated by pharmaceutical companies. The industry rate of success was based on broad categories such as "cancer" or "autoimmunity"; it did not contain any indication specific data for pass rate analysis.

Based on the analysis done in this paper, it is important to note that molecule type impacts the clinical trial success rate of a drug. In addition, unlike other therapeutic areas, small molecules are more successful than biologics in clinical trials for type-2 diabetes. When designing a clinical trial, it is important to note that patient selection is of the utmost importance, because clinical trials conducted with treatment naïve patients are substantially less successful than clinical trials that enrolled treatment naïve patients. Currently, the most successful drug class is the DPP-4 inhibitor drug class, which shows the best risk profile with the previous phase providing adequate risk mitigation for the subsequent phase. At this time, the GLP-1 receptor agonist and SGLT-2 inhibitor drug class lag behind in clinical trial success rate.

\section{AUTHORS CONTRIBUTIONS}

E.A. wrote the manuscript, researched and analyzed the data. J.S. reviewed/edited the manuscript. S.A. assisted with research and data analysis. J.P. designed the study and reviewed/edited the manuscript.

\section{CONFLICT OF INTEREST DISCLOSURE}

Parker consults for investment firms that may or may not have investments owning products in this study. Aiyere works as part of AstraZeneca's Scientific Affairs organization. Ali works as part of AstraZeneca's MedImmune research development organization.

\section{REFERENCES}

1. Bloomgarden, Z. T. "Type 2 Diabetes in the Young: The Evolving Epidemic." Diabetes Care 27.4 (2004): 998-1010.

2. Wild, S., G. Roglic, A. Green, R. Sicree, and H. King. "Global Prevalence of Diabetes: Estimates for the Year 2000 and Projections for 2030." Diabetes Care 27.5 (2004): 1047-053.

3. Diabetes Report Card 2012." Diabetes Report Card. National Center for Chronic Disease Prevention and Health Promotion, 2012. Web. 20 Feb. 2013.

4. National Center for Chronic Disease Prevention and Health Promotion. "Diabetes Report Card 2012." (2012).

5. American Diabetes Association. "Standards of Medical Care in Diabetes - 2013." Diabetes Care 36 (2013): S11-66.

6. Inzucchi, Silvio E., Richard M, Bergenstal, John B. Buse, Michaela Diamant, Ele Ferrannini, Michael Nauck, Anne L. Peters, Apostolos Tsapas, Richard Wender, and David R. Matthews. "Management of Hyperglycemia in Type 2 Diabetes: A PatientCentered Approach." Diabetes Care 35 (2012): 1364-379.

7. Brown, J. B., C. Conner, and G. A. Nichols. "Secondary Failure of Metformin Monotherapy in Clinical Practice." Diabetes Care 33.3 (2010): 501-06. 
8. Husten, Larry. "FDA Approves First SGLT2 Inhibitor For Diabetes." Forbes. Forbes Magazine, 29 Mar. 2013. Web. 30 Oct. 2013.

9. Scheen, André J., and Régis P. Radermecker. "Addition of Incretin Therapy to Metformin in Type 2 Diabetes." The Lancet 375.9724 (2010): 1410-412.

10. Jabbour, S. A., and B. J. Goldstein. "Sodium Glucose Co-transporter 2 Inhibitors: Blocking Renal Tubular Reabsorption of Glucose to Improve Glycaemic Control in Patients with Diabetes." International Journal of Clinical Practice 62.8 (2008): 1279-1284.

11. Osborne BJ, Kaul R, Parker JL. Drug development risk in HIV-1 clinical trials: The effect of drug class. J Pharmaceut Health Serv Res 2011;2:211-6.

12. Falconi A, Lopes G and J.L. Parker. Biomarker and Receptor Targeted therapies reduce Clinical Trial Risk in Non-Small Cell Lung Cancer. Journal of Thoracic Oncology (in press).

13. Parker JL, Kohler JC. The success rate of new drug development in clinical trials: Crohn's disease. J Pharm Pharm Sci 2010;13:191-7.

14. Parker JL, Zhang ZY, Buckstein R. Clinical trial risk in non-Hodgkin's lymphoma: Endpoint and target selection. J Pharm Pharm Sci 2011;13:227-35.

15. Tenuta, A., L. Klotz, and J. L. Parker. "Clinical Trial Risk in Castration Resistant Prostate Cancer: Immunotherapies Show Promise." British Journal of Urology International(in press).

16. Jayasundara, Kavisha S., Edward C. Keystone, and Jayson L. Parker. "Risk of Failure of a Clinical Drug Trial in Patients with Moderate to Severe Rheumatoid Arthritis." The Journal of Rheumatology 39.11 (2012): 2066-070.

17. Amylin and Lilly Announce FDA Approval of BYETTA(TM) (Exenatide) Injection." -- NULL. PR Newswire, 29 Apr. 2005. Web. 30 Oct. 2013.

18. Januvia: "News \& Events." FDA Approves New Treatment for Diabetes. U.S Food and Drug Administration, 17 Oct. 2006. Web. 30 Oct. 2013.

19. Onglyza: "Saxagliptin Approval: Finally,
Competition for Merck's Januvia." Health Blog RSS. The Wall Street Journal, 31 Jan. 2009. Web. 30 Oct. 2013.

20. Victoza: "News \& Events." FDA Approves New Treatment for Type 2 Diabetes. U.S Food and Drug Administration, 25 Jan. 2010. Web. 30 Oct. 2013.

21. Tradjenta: "News \& Events." FDA Approves New Treatment for Type 2 Diabetes. U.S Food and Drug Administration, 2 May 2011. Web. 30 Oct. 2013.

22. Liscinsky, Morgan. "U.S. Food and Drug Administration." FDA Approves Three New Drug Treatments for Type 2 Diabetes. N.p., 25 Jan. 2013. Web. Fall 2013.

23. Liscinsky, Morgan. "U.S. Food and Drug Administration." FDA Approves Farxiga to Treat Type 2 Diabetes. U.S. Food and Drug Administration, 08 Jan. 2014. Web. 08 Jan. 2014.

24. DiMasi, J. A., L. Feldman, A. Seckler, and A. Wilson. "Trends in Risks Associated With New Drug Development: Success Rates for Investigational Drugs." Clinical Pharmacology \& Therapeutics 87.3 (2010): 272-77.

25. Schellekens, H. "Immunogenicity of Therapeutic Proteins: Clinical Implications and Future Prospects." Clinical Therapeutics 24.11 (2002): 1720-740.

26. Weber, Richard W. "Adverse Reactions to Biological Modifiers." Current Opinion in Allergy and Clinical Immunology 4.4 (2004): 277-283.

27. Parker JL, Lushina N, Bahl P, Petrella T, Dent R, and Lopez G. Impact of biomarkers on clinical trial risk in breast cancer. Breast cancer Research and Treatment 136 (2012):179-85.

28. Reid, Geoffrey GJ, Tarek A. Bin Yameen, and Jayson L. Parker. "Impact of Biomarkers on Clinical Trial Risk." Pharmacogenomics 14.13 (2013): 1645658.

29. Reid, Timothy. "Choosing GLP-1 Receptor Agonists or DPP-4 Inhibitors: Weighing the Clinical Trial Evidence." Clinical Diabetes 30.1 (2012): 3-12. 